\title{
Tertiary Biomaterial Encapsulation Controls the Release of FGF-2 without Impacting Bioactivity
}

\author{
Drew Kuraitis ${ }^{\mathrm{a}, \mathrm{b}, \#}$, Zahra Arzhangi, ${ }^{\mathrm{a}, \mathrm{H}}$, Alex J. Hyatta ${ }^{\mathrm{a}}$, Branka Vulesevic ${ }^{\mathrm{a}, \mathrm{b}}$, Kimberley Merrett ${ }^{\mathrm{a}, \mathrm{c}}$, Jin \\ Zhang $^{\mathrm{a}, \mathrm{d}}$, Erik J. Suuronen ${ }^{\mathrm{a}, \mathrm{b}, \#, *}$ and May Griffith ${ }^{\mathrm{a}, \mathrm{c}, \#, *}$
}

\author{
${ }^{a}$ Department of Cellular and Molecular Medicine, University of Ottawa, Ottawa, Canada \\ ${ }^{b}$ University of Ottawa Heart Institute, University of Ottawa, Ottawa, Canada \\ ${ }^{c}$ Integrative Regenerative Medicine Centre, Linköping University, Linköping, Sweden \\ ${ }^{d}$ Department of Chemical and Biochemical Engineering, University of Western Ontario, London, Canada
}

\begin{abstract}
Diseases that restrict the flow of blood to muscles in the peripheral limbs or the heart remain prevalent causes of reduced quality of life and death in developed countries. Signaling molecules that play a role in the regenerative responses are currently being exploited as potential therapies to restore blood flow and tissue function. Fibroblast growth factor-2 (FGF-2) is a potent stimulator of neovascularization. It is believed that a controlled release of a delivered cytokine is superior to a bolus administration for achieving the desired regenerative effect. Therefore, we incorporated FGF-2-containing microspheres into a hydrogel and further encased this hydrogel into a collagen capsule for implantation. Cytokine release was controlled and constant over a month-long study period, and FGF-2 released from this tertiary encapsulation system maintained its bioactivity, as measured by its proliferative effects on endothelium. In a subcutaneous mouse model, FGF-2 treatment induced a systemic response that included increased stem cell chemoattractant cytokines, the mobilization of a potent $\mathrm{CXCR} 4^{+}$angiogenic population, and also an increase in the density of small diameter blood vessels. These observations were accompanied by no changes in the systemic levels of inflammatory cytokines. Overall, tertiary encapsulation of FGF-2 retards its release and allows for a more controlled and constant delivery of FGF-2, while maintaining its bioactive effects on endothelial cells and systemic responses in vivo.
\end{abstract}

Keywords: Controlled Release, Cytokines, Encapsulation, Hydrogel, Fibroblast Growth Factor, Neovascularization.

\section{INTRODUCTION}

Two of the most prevalent diseases that continue to place huge financial burdens and reduce the quality of life of individuals living in developed nations are peripheral artery disease [1] and coronary artery disease [2]. Both of these diseases manifest as occlusions of vasculature, which reduce perfusion in peripheral limb [3] or cardiac [4] muscle, leading to poor health, reduced quality-of-life, or even death. Many regenerative therapy strategies are currently being investigated with the aim of restoring perfusion and function to ischemic muscle [5]. A large proportion of these regenerative therapies are investigating the application of stem cells, delivered to the target muscle to induce tissue regeneration. It appears that perhaps the main mechanism responsible for the improvements achieved with stem cell transplantation is that of a paracrine effect [6]. To this end, stem cells may orchestrate regenerative responses by controlling the production and action of small signaling molecules, or cytokines. Since stem cell therapy is associated

*Address correspondence to these authors at the Integrative Regenerative Medicine Centre, Linköping University, Cell Biology Building, Level 10, SE-581 83 Linköping, Sweden; Tel: 46 (0)13-28 17 56;

E-mail: may.griffith@ liu.se and Division of Cardiac Surgery, University of Ottawa Heart Institute, 40 Ruskin Street, Ottawa, ON K1 Y 4W7, Canada;

Tel: 613-798-5555, Ext. 19087; E-mail: esuuronen@ottawaheart.ca

\#denotes equal contributions with some limitations, such as cell availability, immunogenicity, so-far modest results [7], and rapid loss of transplanted cells [8]; another approach would be to mimic the signaling axes that are activated by transplanted stem cells. In this regard, the argument can be made that an acellular approach may allow for improved control over these signaling axes, since cytokine responses from transplanted stem cells vary over time [9] and it cannot yet be relied upon that stem cells will persist in the recipient tissue.

The cytokine fibroblast growth factor-2 (FGF-2) is an excellent candidate to augment vascular regenerative processes. FGF-2 signals for functional bioactivity in vascular cells, such as endothelial proliferation [10] and migration [11]. FGF-2 has been shown to significantly increase the formation of microvascular networks in ischemic myocardium [12]; however, direct introduction of FGF-2 into a system may lead to rapid diffusion [13]. Administered FGF-2 is also rapidly cleared from the body $[14,15]$. The controlled delivery of FGF-2 has been shown to lead to greater microvessel density in vivo [16]; therefore, an approach to control the release of FGF-2 may be superior in order to prolong the cytokine's beneficial effects.

The objectives of this study were two-fold: 1) to assess the potential of secondary and tertiary encapsulation systems of FGF-2 for controlled release; and 2) to assess the 
biocompatibility and use of such a tertiary encapsulation system for the delivery of FGF-2 in vivo.

\section{METHODS}

All materials and reagents were obtained from SigmaAldrich (St. Louis, USA) unless otherwise stated.

\section{FGF-2-loaded Alginate Microparticles (Primary Encapsulation)}

A $2 \%(\mathrm{w} / \mathrm{v})$ solution of alginic acid sodium salt was prepared in water and sterilized by autoclaving. Human recombinant FGF-2 $(10 \mu \mathrm{g}$; BD Biosciences, Mississauga, Canada) was reconstituted in a $1 \mathrm{ml}$ PBS solution containing $0.1 \%$ bovine serum albumin. Alginate was added to the FGF-2 solution in a 9:1 (v/v) ratio, and allowed to thoroughly mix with gentle agitation at room temperature. The FGF-2-alginate solution was loaded into a $1 \mathrm{ml}$ syringe and sprayed from a height of $14 \mathrm{~cm}$ into a bath of $2.5 \%$ (w/v) cross-linking $\mathrm{CaCl}_{2}$ using a spray gun connected to an air compressor, propelled by a constant stream of $\mathrm{N}_{2}$ gas. After 20 minutes of Ca-induced gelation, the Ca-alginate particles were collected, rinsed twice and resuspended in water. Particles were generated with FGF-2 and without FGF-2 (blank) incorporation and were subsequently stored at $-80^{\circ} \mathrm{C}$ until used.

Stereomicroscopic images of hydrated particles were taken (Nikon Eclipse TE2000-E, Nikon, Mississauga, Canada) and particle diameters were measured using Northern Eclipse software. Representative samples were also mounted on metal holders and sputter-coated with a gold layer for 60 seconds at 0.1 bar vacuum pressure (Sputter Coater 108, Cressington, Watford, UK) prior to examination by scanning electron microscopy (SEM; Model S-2250N, Hitachi, Tokyo Japan).

\section{Collagen Hydrogel (Secondary Encapsulation)}

A collagen hydrogel was produced using a procedure modified from Yang et al. [17]. Briefly, rat type I collagen $3.45 \mathrm{mg} / \mathrm{ml}$ (BD Biosciences, Mississauga, Canada) was mixed in ice water $\left(4^{\circ} \mathrm{C}\right)$ with a buffered serum-free medium $\left(10 \times \mathrm{M} 199: 7.5 \% \mathrm{NaHCO}_{2}\right.$ : 1M HEPES: water; 10:1:1:8) in a ratio of $8: 2(\mathrm{v} / \mathrm{v})$. The $\mathrm{pH}$ of the collagen-buffer mixture was then adjusted to 7.0 by titration with $1 \mathrm{M} \mathrm{NaOH}$. Genipin solution $(2 \mathrm{mg} / \mathrm{ml}$; Wako Chemicals, Osaka, Japan) was added to the buffered collagen solution to a final concentration of $0.05 \mathrm{mg} / \mathrm{ml}$ and the collagen solution was mixed again on ice. For particle incorporation, particles suspended in water were added to the hydrogel in a ratio of $2: 8(\mathrm{w} / \mathrm{v})$. Crosslinking of the hydrogels by genipin was assessed by differential scanning calorimetry (DSC Q2000 instrument, TA Instruments, Denmark).

\section{Collagen Capsules (Tertiary Encapsulation)}

An aqueous porcine type I atelocollagen $(10 \% \mathrm{w} / \mathrm{w})$ (Nippon Ham, Japan) was prepared in $\mathrm{ddH}_{2} \mathrm{O}$ within a syringe mixing system as per Liu et al. [18]. An empty gelatin capsule (No. 4; T.U.B. Enterprises, Almonte, Canada) was coated with $100 \mu$ of the collagen solution (pre-warmed to $37^{\circ} \mathrm{C}$ in a humidified incubator to decrease viscosity) and subsequently cross-linked with $\mathrm{N}$-(3dimethylaminopropyl)-N'-ethylcarbodiimide hydrochloride
(EDC) and N-hydroxysuccinimide (NHS) in a 2:1 (w/w) ratio. This reaction proceeded at a $\mathrm{pH}$ range of 3-5. The capsule was then washed twice with water and stored overnight at $4^{\circ} \mathrm{C}$ in $1 \%$ chloroform ( $\mathrm{v} / \mathrm{v}$; in PBS) to maintain sterility. Daily washes in PBS were conducted for 5 subsequent days to remove residual chloroform.

\section{Loading Capsules (Tertiary Encapsulation)}

Microparticles were mixed into the collagen hydrogels at a ratio of $2: 8(\mathrm{w} / \mathrm{v})$ and injected into collagen capsules using a 16-gauge $1 \frac{1}{2}$ inch needle. Each capsule delivery system was sealed inside a 12-well Costar ${ }^{\circledR}$ Transwell plate (Corning, New York, USA) and placed in a humidified incubator overnight at $37^{\circ} \mathrm{C}$ for curing to occur.

\section{Immortalized Human Umbilical Vein Endothelial Cell (IHUVEC) Culture}

IHUVECs were grown in $25 \mathrm{~cm}^{2}$ tissue culture flasks (Corning Inc.) coated with $1 \%$ gelatin and supplemented with growth medium M199 (Invitrogen, Burlington, Canada) containing $10 \%$ heat-inactivated fetal bovine serum (HIFBS), $90 \mathrm{mg} / \mathrm{L}$ heparin, $50 \mu \mathrm{g} / \mathrm{mL}$ endothelial cell growth supplement (ECGS) and $5 \mu \mathrm{g} / \mathrm{mL}$ gentamicin (Invitrogen). Cells were maintained in a humidified incubator at $37^{\circ} \mathrm{C}$ and $5 \% \mathrm{CO}_{2}$, until $80 \%$ confluence. Trypsin-ethylenediaminetetraacetic acid (Trypsin-EDTA; Invitrogen) was used to detach the cells from the substrate, after rinsing with $\mathrm{Ca}^{2+}$ and $\mathrm{Mg}^{2+}$-free $0.1 \mathrm{M}$ phosphate buffered saline (PBS). Cells were incubated for 2 minutes with $0.05 \%$ Trypsin-EDTA, after which the reaction was terminated by adding serumcontaining M199. Dislodged cells were then centrifuged at $1500 \mathrm{rpm}$ for 5 minutes, resuspended in M199 containing $2 \%$ HI-FBS without ECGS to obtain a concentration of 150,000 cells $/ \mathrm{mL}$.

\section{FGF-2 Release}

Enzyme-linked immunosorbent assays (ELISA; R\&D Systems Inc., Minneapolis, USA) were used to quantify the amount of FGF-2 released from the following three systems: 1) FGF-2 alginate microparticles only; 2) collagen hydrogels containing FGF-2 alginate microparticles; and 3) capsules loaded with collagen hydrogel containing FGF-2 alginate microparticles. All samples were incubated in growth factorfree M199 medium (Invitrogen) at $37^{\circ} \mathrm{C}$. Supernatant was collected and replaced with fresh medium daily, for 29 days. Collected samples were stored at $-20 \mathrm{C}$ and the concentration of released FGF-2 was quantified using ELISA, as per the manufacturer's instructions $(n=3)$.

\section{WST-1 Assay for FGF-2-Induced Bioactivity}

Daily-released FGF-2 supernatant samples $(50 \mu \mathrm{L})$ were added to HUVECs pre-seeded $(7,500$ cells/well) in $2 \%$ FBS M199 media $(50 \mu \mathrm{L})$ in a 96 -well plate. For the normalization assay, daily-released FGF-2 supernatant samples were diluted to a normalized concentration $(0.3 \mathrm{ng} / \mathrm{mL})$ using $2 \%$ FBS M199 media. Non-encapsulated, exogenously added FGF-2 $(0.3 \mathrm{ng} / \mathrm{mL})$ in 2\% FBS M199 and 2\% HI-FBS M199 media alone were used as positive and negative controls, respectively. After incubation of the cells for a period of $72 \mathrm{~h}$, $10 \mu 1 /$ well of the reagent WST-1 (Roche, Indianapolis, USA) was added and incubated for $1 \mathrm{~h}$ at $37^{\circ} \mathrm{C}$. The dye was quantified by measuring the optical density at $450 \mathrm{~nm}$ with a 


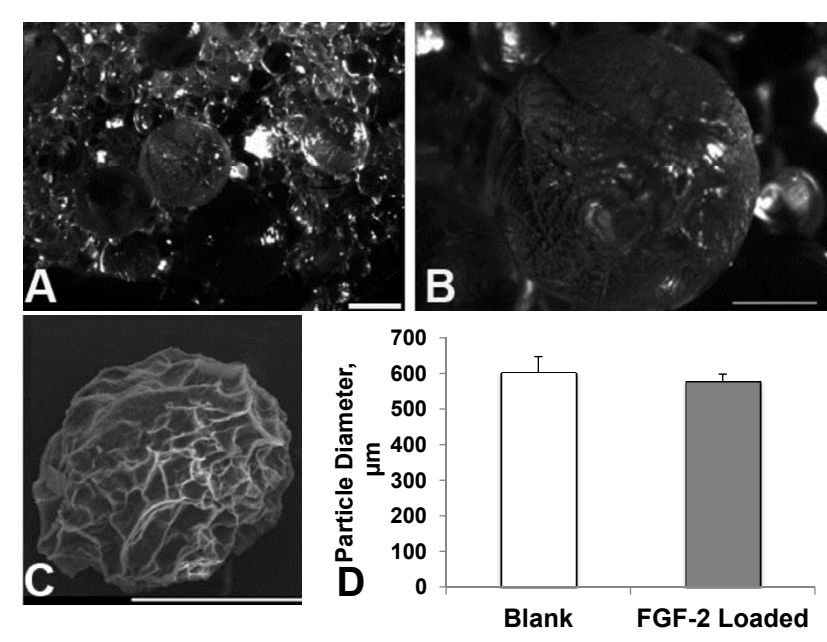

Fig. (1). Morphology of alginate microparticles. Particles vary in size and present a smooth surface $(\mathbf{A}$; scale bar $=1000 \mu \mathrm{m})$. Greater magnification reveals a highly textured surface $(\mathbf{B}$; scale bar $=500 \mu \mathrm{m})$. SEM micrograph $(\mathbf{C})$ allows for detailed visualization of the lattice-like folds and protrusions on the microparticles (scale bar $=200 \mu \mathrm{m}$ ). Incorporation of FGF-2 did not affect the size of microparticles produced $(p=0.9 ; \mathbf{D})$.

scanning multi-well spectrophotometer (Bio-Tek Instruments Inc., Burlington, USA). Media and WST-1 dye were aspirated after microplate reading and plates were stored at $20^{\circ} \mathrm{C}$. Fold-changes were calculated using the optical densities at $450 \mathrm{~nm}$ in a ratio of samples containing FGF-2 alginate particles:blank alginate particles $(n=4)$.

\section{CyQUANT Cell Proliferation Assay}

Similarly to the WST-1 assay, samples containing released FGF-2 over time were collected. The same experiment was performed, but with pooled supernatant and the CyQUANT Cell Proliferation Assay (Molecular Probes, Eugene, USA), according to the manufacturer's instructions. Fluorescence was measured directly on a microplate fluorescence reader (Bio-Tek Instruments Inc.) with excitation at $485 \mathrm{~nm}$ and emission detection at $530 \mathrm{~nm}$. Foldchanges were calculated using the fluorescence signals in a ratio of samples containing FGF-2 alginate particles:blank alginate particles $(n=4)$.

\section{FGF-2 Delivery Study}

A subcutaneous implant mouse model was used to investigate the efficacy of collagen capsules for FGF-2 delivery. Animal surgeries were performed with the approval of the University of Ottawa Animal Care Committee, in accordance with the National Institute of Health's Guide for the Care and Use of Laboratory Animals. All procedures were performed under $2 \%$ isoflurane anesthesia. Nude BALB/c mice (3-weeks old; Jackson Laboratories, Bar Harbour, USA) received two subcutaneous dorsal implants of collagen capsules containing hydrogel with blank or FGF2-loaded alginate microparticles, as described [19]. After 3 weeks, animals were anesthetized and euthanized via cardiac puncture. Blood from the termination procedure was collected and spun at $2000 \mathrm{~g}$ for 30 minutes to obtain serum. Serum samples were stored at $-80^{\circ} \mathrm{C}$. Implants were dissected and prepared for histology as described below $(n=5)$.

\section{Flow Cytometry}

As previously described [20], blood samples $(50-100 \mu 1)$ were procured from the saphenous veins prior-to surgery and after 1 and 2 weeks. Peripheral blood mononuclear cells were isolated using density-gradient centrifugation. Cells were labeled with anti-CXCR4 (BD Biosciences, Mississauga, Canada) and analyzed with a FACSAria flow cytometer (BD Biosciences). The isotype-matched immunoglobulin antibody (BD Biosciences) was used as a control $(n=5)$.

\section{Cytokine Arrays}

As per the manufacturer's instructions, Mouse Cytokine Antibody Arrays (Raybiotech, Norcross, USA) were used to assess relative levels of systemic cytokines in mouse serum. Serum was thawed on ice and $10 \mu \mathrm{l}$ was diluted to $1 \mathrm{ml}$ with the supplied diluent before incubation on array membranes. Data was quantified using AlphaEaseFc, and is reported as a ratio of cytokine:total protein $(n=5)$.

\section{Histology}

Implants were removed from euthanized mice and were fixed overnight in 4\% paraformaldehyde, then subsequently dehydrated and paraffin-embedded. Sections were blocked with $10 \%$ hydrogen peroxide in water for $5 \mathrm{~min}$ to block endogenous peroxidase activity. Antigen retrieval was performed by microwave treatment of the sections in citrate buffer for $10 \mathrm{~min}$, followed by blocking of with normal rabbit serum (1:5). CD31 staining was performed using rabbit polyclonal anti-CD31 (1:100; Abcam, Cambridge, USA) followed by biotinylated secondary anti-rabbit antibody $(1: 1000)$ for $45 \mathrm{~min}$. After incubation in avidin-biotin peroxidase complex for 30min, 3,3-diaminobenzidine tetrahydrochloride (DAB) was applied. Between steps, the slides were rinsed for $5 \mathrm{~min}$ in PBS three times. Sections were then counterstained with haematoxylin for $15 \mathrm{sec}$, dehydrated, and mounted. Images were captured on an Axioskop light microscope (Zeiss, Toronto, Canada) with an AxioCam camera (Zeiss) and CD31-positive vessels were quantified by using ImageJ software $(n=4)$.

\section{Statistical Analysis}

All in vitro values are given as mean \pm standard deviation (SD) from at least three independent experiments. Continuous data was analyzed with a one-way ANOVA and a two-tailed Student's $t$-test was used when comparing the mean values of each conducted experiment, unless otherwise indicated. Values of $p<0.05$ were considered significant.

\section{RESULTS}

\section{Physical Characterization of FGF-2-Loaded Microparticles}

Upon contact with $\mathrm{CaCl}_{2}$ solution, alginate droplets formed soft gel beads. Representative stereo micrograph images revealed Ca-alginate particles of various sizes with a glossy surface appearance (Fig. 1A). Higher magnification of larger particles revealed a highly textured surface (Fig. 1B). The majority of particles (98\%) were within the micrometer range (data not shown); therefore alginate particles were referred to as microparticles. For future applications, the freeze-drying of FGF-2 encapsulated 


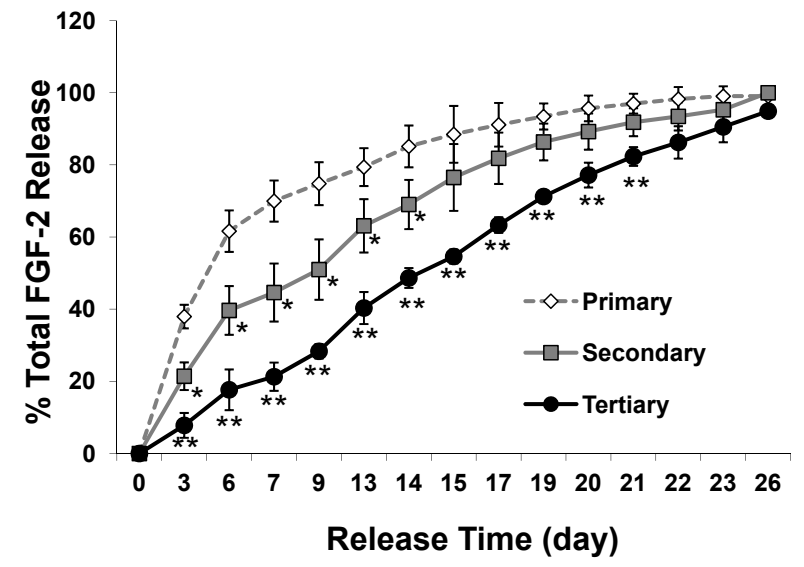

Fig. (2). Release effects of primary, secondary and tertiary FGF-2 encapsulation. Tertiary encapsulation prolonged FGF-2 release up to 21 days (**p<0.05 vs. both primary and secondary systems) and secondary encapsulation also had a retarding effect on FGF-2 release, up to day $14\left({ }^{*} p<0.05\right.$ vs. primary system).

alginate microparticles would be ideal for their preservation post-production [21]. Therefore, SEM analysis was performed to compare the surface structures of alginate particles post freeze-drying versus storage at $-80^{\circ} \mathrm{C}$. SEM imaging revealed preservation of particle shape and surface texture (Fig. 1C). FGF-2 incorporation did not affect the alginate microparticle size ( $p=0.09$; Fig. 1D).

\section{Secondary and Tertiary Encapsulation Prolongs FGF-2 Release}

It is desirable to maintain a controlled release of encapsulated cytokines. Primary encapsulated FGF-2 loaded into $\mathrm{Ca}$-alginate microspheres alone displayed a burst-release effect, with most of the growth factor released into the surrounding environment in less than 9 days (Fig. 2). Secondary encapsulation into a genipin crosslinked collagen hydrogel may have slightly reduced the burst effect, but the majority of FGF-2 was still released in a similar time frame (Fig. 2). The most profound effect on FGF-2 release was its tertiary encapsulation, achieved by further incorporation into a collagen capsule; in this system, FGF-2 release appears constant for up to 29 days of observation (Fig. 2). Statistically, FGF-2 was released from the tertiary system in lesser amounts over time, until day $21(p<0.05)$. Secondary hydrogel encapsulation also retarded the release of FGF-2, although to a lesser degree, until day $14(p<0.05)$.

\section{Bioactivity of Released FGF-2}

FGF-2 is known to be an initiator of endothelial cell proliferation [11]. To ensure that FGF-2 handling and microparticle and hydrogel incorporations did not negatively impact its bioactivity, the released FGF-2 was assessed for its ability to stimulate IHUVEC proliferation. Using WST (Fig. 3A) and CyQuant (Fig. 3B) methods, released FGF-2 could stimulate a $36 \%$ increase in cell proliferation $(P<0.05)$. Additionally, an average increase of $35 \%$ in proliferation was observed when released FGF-2 collected from days 3, 9, 15, 21 and 24 was added to IHUVEC cultures (Fig. 3C; $P<0.05)$, demonstrating that bioactivity of FGF-2 did not change with the duration of encapsulation. These
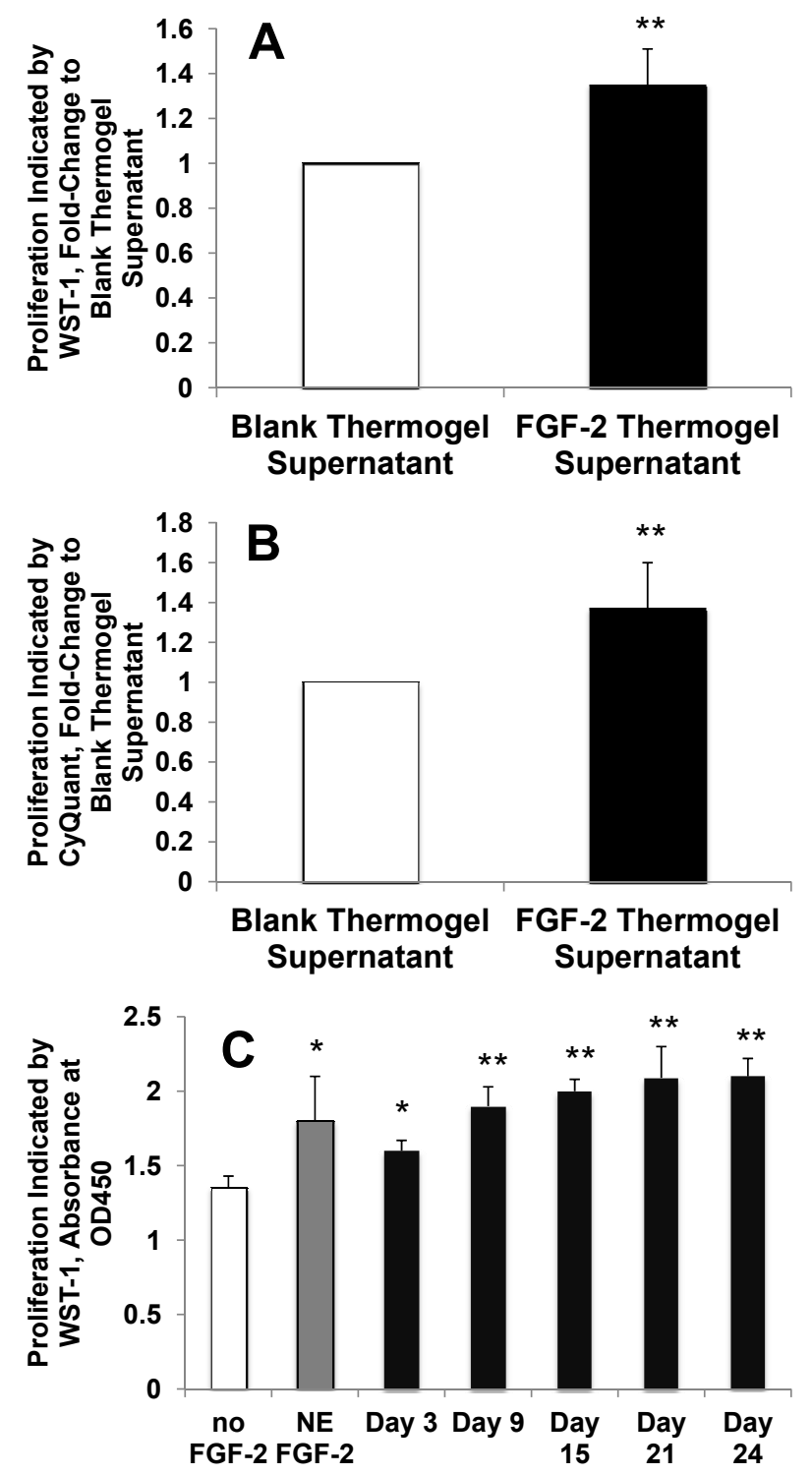

Fig. (3). Bioactivity of FGF-2 released from secondary and tertiary encapsulation systems. IHUVECs incubated with FGF-2-containing supernatant collected from hydrogels-containing FGF-2-loaded microparticles were observed to proliferate, measured by WST-1 (A) and CyQuant (B), compared to supernatant of a parallel system without FGF-2 incorporation. Further encapsulation into a tertiary capsule induced a similar effect $(\mathbf{C})$, compared to controls without FGF-2. There was no difference in bioactivity between nonencapsulated (NE) FGF-2 and FGF-2 released from the tertiary system between days $3-24$. Supernatant was added so that the amount of FGF-2 was consistently $0.30 \mathrm{ng} / \mathrm{ml}$. $* P<0.05$; $* * P<0.01$.

observations were not different from the addition of non-encapsulated, native FGF-2 at the same concentration (Fig. 3C).

\section{Tertiary Encapsulation of FGF-2 Systemically Mobilizes Progenitor Cells}

FGF-2 that was incorporated into three systems (microparticles + hydrogel + capsule) further maintained bioactivity in vivo. Compared to FGF-2-free implants, the loaded capsules were able to stimulate a mobilization of 


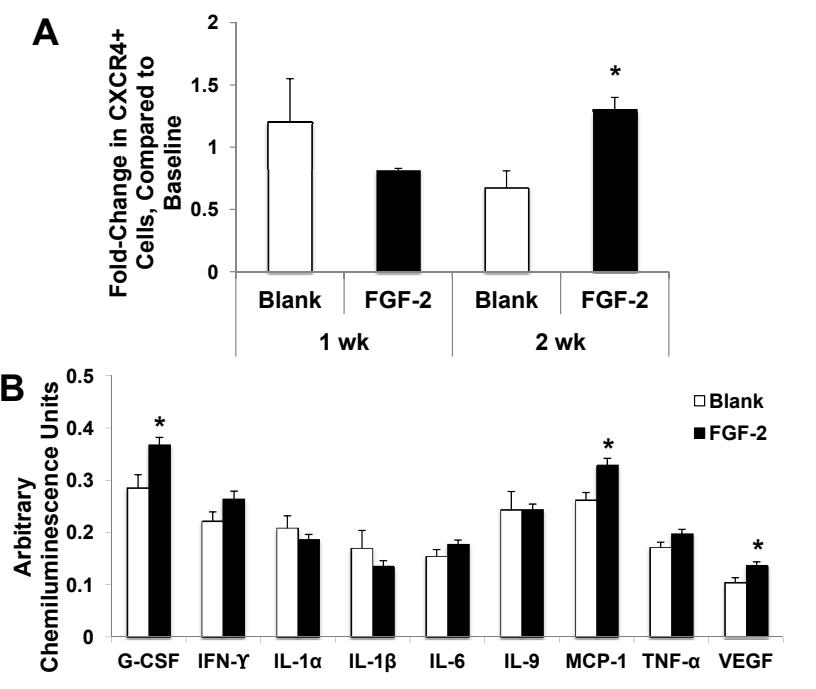

Fig. (4). Systemic effects in mice resulting from subcutaneous implantation of FGF-2 tertiary encapsulation system. At 2 weeks post-implantation, there was an increase in the number of circulating $\mathrm{CXCR}^{+}$cells (A; $p=0.03$ ). FGF-2 incorporation increased systemic levels of chemotactic cytokines G-CSF $(p=0.03), \operatorname{MCP}-1 \quad(p=0.01)$ and VEGF $(p=0.04)$ and induced no changes in the levels of the inflammatory cytokines IFN- $\gamma$, IL- $1 \alpha$, $\beta,-6,-9$ or TNF- $\alpha(\mathbf{B})$.

$\mathrm{CXCR}^{+}$cells into the circulation. Cells were monitored at 1and 2-week post-implantation. There was no difference between circulating $\mathrm{CXCR}^{+}$cells at 1 week (Fig. 4A; $P=0.3$ ), but at 2 weeks, there was a $94 \%$ increase with FGF-2 treatment (Fig. 4A; $P=0.003$ ).

\section{Tertiary Encapsulation of FGF-2 Increases Serum Levels of Therapeutic Cell-Recruiting Cytokines}

At sacrifice, the effects of FGF-2 treatment on serum cytokine levels were apparent. There were increased serum levels of granulocyte-colony stimulating factor (G-CSF) by 29\% (Fig. 4B; $P=0.030$ ), monocyte chemoattractant protein1 (MCP-1) by $25 \%$ (Fig. 4B; $P=0.013$ ) and vascular endothelial growth factor (VEGF) by $31 \%$ (Fig. 4B; $P=0.045$ ). There were no observable differences between the inflammatory cytokines interferon- $\gamma$, interleukin (IL)- $1 \alpha$, $1 \beta,-6,-9$, or tumor necrosis factor- $\alpha$ (TNF- $\alpha$; Fig $4 \mathbf{B}$; all $P>0.15)$.

\section{FGF-2-Containing Capsules Are Permissive to Vascular Growth}

Immunostaining against CD31 was performed to identify vascular structures. $\mathrm{CD} 31^{+}$cells arranged in vessel-like shapes were visible and in abundance surrounding the capsule (Fig. 5A). There was a 3.1-fold increase in the relative number of small diameter vessels $(<250$ pixels; $p=0.04$ ) surrounding FGF-2-containing capsules, compared to capsules without FGF-2 (Fig. 5B); however, there was no difference in the relative number of larger diameter vessels $(p \geq 0.3)$. Overall, signs of host vascularization and capsule compatibility were observed.

\section{DISCUSSION}

Delivery of small peptides in microparticles is now routinely used clinically. Many methods have been

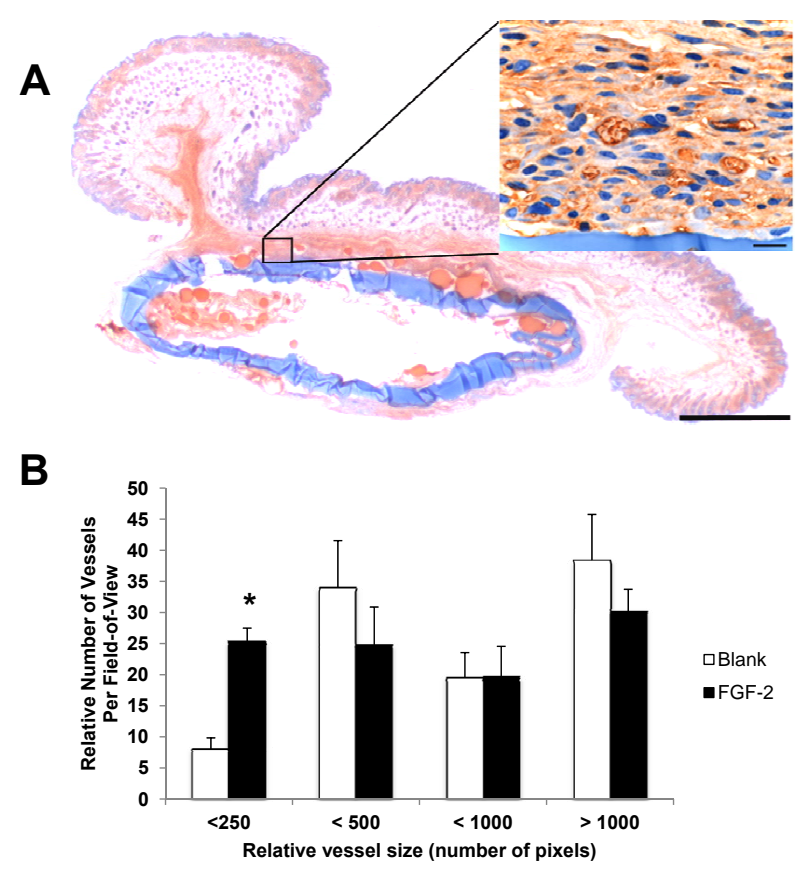

Fig. (5). Immunostaining of $\mathrm{CD} 31^{+}$structures surrounding the implant. By 3 weeks, the capsule implant had integrated into the tissue and was adherent to the dermal layers (A). CD $31^{+}$vessels could be seen in the tissue immediately surrounding the capsule, visualized by DAB staining (dark brown). There was a 3.1-fold increase in the relative number of small diameter vessels (B); however, no differences in vessels of larger diameters were observed between treatments. $* P=0.04$; scale bar $=5000 \mu \mathrm{m}$.

developed to control the release characteristics of the encapsulated peptides, including the use of core-shell particles and coating of the microspheres to sustain release. In this study, we tested the feasibility of extending peptide release from microspheres from within a hydrogel construct. We show that: 1) FGF-2-encapsulated alginate microparticles may be stored by freezing at $-80^{\circ} \mathrm{C}$, without negative impact on peptide bioactivity; 2) secondary encapsulation can delay peptide release, but tertiary encapsulation provides a superior controlled release profile, while still maintaining bioactivity; and 3) FGF-2 delivered in this manner can increase systemic progenitor mobilizing cytokines, induce the mobilization of circulating angiogenic cells, and also support neovascularization.

The hydrogels used in this experiment gelled rapidly, with their preparation taking only minutes. However, the protocols for generating alginate microparticles may require longer periods. Therefore, it is advantageous, especially with a clinical application in mind, that the microparticles can be stored via freeze-drying. This would allow for their rapid incorporation into the gel materials prior to application under time constraints associated with surgery.

Secondary encapsulation into an injectable hydrogel appears to mildly prolong the burst release of FGF-2 from the microparticles. A similar model has also shown this effect of reduced burst release effect, but using stromal cellderived factor-1-containing alginate microparticles and an injectable collagen-chondroitin sulfate hydrogel[20]. Notably, in the current study, tertiary encapsulation into a 
collagen capsule equilibrated the release and led to more stable and sustained release over the month-long study period. By inherent design, many delivery systems suffer from an initial burst release, whereby a relatively large amount of therapeutic agent is released in a disproportionately rapid amount of time [22-24]. Studies that have incorporated FGF-2 into polymer-releasing systems have noted burst-release effects [25-27]. Since administered FGF-2 has a relatively short half-life in the body, it would be advantageous to have a controlled presence of FGF-2 to elicit constant effects. Additionally, potential sources of peptide bioactivity loss during implant preparation (frozen storage and chemical cross-linking) failed to reduce the ability of released FGF-2 to stimulate the proliferation of endothelial cells, highlighting the compatibility of the presented delivery system.

A subcutaneous in vivo model was used to evaluate biocompatibility and systemic effects of FGF-2 delivered in a tertiary encapsulation system. One week after implantation, there were no apparent differences in circulating $\mathrm{CXCR}^{+}$ progenitor cells; however, at two weeks, animals receiving the treatment had a marked increase in circulating $\mathrm{CXCR}^{+}$ cells. This bone marrow-derived population of circulating cells has recently been highlighted as having extreme potency for invasion and neovascularization, and to secrete regenerative cytokines in greater amounts, compared to the CXCR4- fraction [28]. FGF-2 has been shown to play an essential role in the mobilization of progenitor cells from the bone marrow to the circulation [29]. Therefore, the increase in $\mathrm{CXCR}^{+}$cell mobilization to the circulation points to the promise of the FGF-2 tertiary encapsulation system as a therapy aimed towards angiogenesis via a systemic effect on recruitment of therapeutic cells of the bone marrow.

Other systemic effects of our FGF-2 delivery system were revealed by cytokine analysis of the serum collected at termination. Animals receiving FGF-2 treatment demonstrated higher levels of therapeutic cell-mobilizing cytokines G-CSF, MCP-1 and VEGF. Other studies have correlated enhanced angiogenesis in animal models with increased levels of G-CSF [30], MCP-1 [19, 31] and VEGF [30]. Based on the serum cytokine profile observed, it may prove to be that another effect of our FGF-2 delivery system is to induce a systemic environment that is supportive of angiogenesis. There were no observed changes in relative levels of inflammatory cytokines (IL- $1 \alpha,-\beta,-6,-9$, IFN- $\gamma$, $\mathrm{TNF} \alpha$ ). In addition, there were no apparent inflammatory reactions observed in the excised collagen capsules, and only a mild amount of fibrous tissue, such that the capsules could easily be separated from the skin. Vasculature was observed from all angles surrounding the capsules, suggesting that the capsules are able to support neovascularization. Given that the survival of an implant is largely dependent on its vascularization [32], observed increases in small-diameter vessels surrounding capsules containing FGF-2 suggest a more rapid stimulation of angiogenesis and integration with the host.

Collagen capsule tertiary encapsulation serves as a method to greatly prolong the release of, and maintain the bioactivity of FGF-2. This biocompatible system is efficacious at activating systemic cell and cytokine responses that have the potential to augment angiogenesis. This study suggests that it not only supports local vascular growth but it has the potential to enhance local angiogenesis while maintaining peptide bioactivity. The establishment of biocompatibility and bioactivity of our tertiary encapsulation system supports future evaluation in disease models to assess efficacy in diseased tissue. Based on these results, an investigation of how much neovascularization the capsules can induce in a model of ischemia would be a foreseeable next step.

\section{CONCLUSIONS}

The conclusions of this study are two-fold. Third-degree encapsulation slows the release of FGF-2, as intended for slow and controlled release of this growth factor, while it did not seem to interfere with its bioactivity. Secondly, the biocompatibility permits local vascular growth, and may potentially induce systemic angiogenesis, as demonstrated by increased chemoattractants and circulating angiogenic cells, and a greater proportion of small-diameter vessels.

\section{CONFLICT OF INTEREST}

The authors have no conflicts of interest to report.

\section{ACKNOWLEDGEMENTS}

This project was funded by a Canadian Stem Cell Network grant and AFA Försäkring Sweden grant (to MG), and by a grant-in-aid T6793 from the Heart \& Stroke Foundation of Canada (to EJS). ZA was supported by an Ontario Graduate Student Science and Technology Scholarship, DK was supported by a CIHR-CGS Doctoral Award. We thank Ms. Abeni Wickham for help with collagen hydrogel characterization.

\section{REFERENCES}

[1] Belch JJ, Topol EJ, Agnelli G, et al. Critical issues in peripheral arterial disease detection and management: a call to action. Arch Intern Med 2003; 163(8): 884-92.

[2] Lloyd-Jones D, Adams R, Carnethon M, et al. Heart disease and stroke statistics--2009 update: a report from the American Heart Association Statistics Committee and Stroke Statistics Subcommittee. Circulation 2009; 119(3): e21-181.

[3] Selvin E, Erlinger TP. Prevalence of and risk factors for peripheral arterial disease in the United States: results from the National Health and Nutrition Examination Survey, 1999-2000. Circulation 2004; 110(6): 738-43.

[4] Worthley SG, Osende JI, Helft G, Badimon JJ, Fuster V. Coronary artery disease: pathogenesis and acute coronary syndromes. Mt Sinai J Med 2001; 68(3): 167-81.

[5] Kuraitis D, Giordano C, Ruel M, Musaro A, Suuronen EJ. Exploiting extracellular matrix-stem cell interactions: A review of natural materials for therapeutic muscle regeneration. Biomaterials 2012; 33(2): 428-43.

[6] Gnecchi M, Zhang Z, Ni A, Dzau VJ. Paracrine mechanisms in adult stem cell signaling and therapy. Circ Res 2008; 103(11): 1204-19.

[7] Kuraitis D, Suuronen EJ, Sellke FW, Ruel M. The future of regenerating the myocardium. Curr Opin Cardiol 2010; 25(6): 57582 .

[8] Suuronen EJ, Kuraitis D, Ruel M. Improving cell engraftment with tissue engineering. Semin Thorac Cardiovasc Surg 2008; 20(2): 110-4.

[9] Cho HJ, Lee N, Lee JY, et al. Role of host tissues for sustained humoral effects after endothelial progenitor cell transplantation into the ischemic heart. J Exp Med 2007; 204(13): 3257-69.

[10] Lindner V, Majack RA, Reidy MA. Basic fibroblast growth factor stimulates endothelial regrowth and proliferation in denuded arteries. J Clin Invest 1990; 85(6): 2004-8. 
[11] Yoshida A, Anand-Apte B, Zetter BR. Differential endothelial migration and proliferation to basic fibroblast growth factor and vascular endothelial growth factor. Growth Factors 1996; 13(1-2): 57-64.

[12] Takehara N, Tsutsumi Y, Tateishi K, et al. Controlled delivery of basic fibroblast growth factor promotes human cardiospherederived cell engraftment to enhance cardiac repair for chronic myocardial infarction. J Am Coll Cardiol 2008; 52(23): 1858-65.

[13] Edelman ER, Mathiowitz E, Langer R, Klagsbrun M. Controlled and modulated release of basic fibroblast growth factor. Biomaterials 1991; 12(7): 619-26.

[14] Bush MA, Samara E, Whitehouse MJ, et al. Pharmacokinetics and pharmacodynamics of recombinant FGF-2 in a phase I trial in coronary artery disease. J Clin Pharmacol 2001; 41(4): 378-85.

[15] Whalen GF, Shing Y, Folkman J. The fate of intravenously administered bFGF and the effect of heparin. Growth Factors 1989; 1(2): 157-64.

[16] Jeon O, Kang SW, Lim HW, Hyung Chung J, Kim BS. Long-term and zero-order release of basic fibroblast growth factor from heparin-conjugated poly(L-lactide-co-glycolide) nanospheres and fibrin gel. Biomaterials 2006; 27(8): 1598-607.

[17] Yang B, Cao DJ, Sainz I, Colman RW, Guo YL. Different roles of ERK and p38 MAP kinases during tube formation from endothelial cells cultured in 3-dimensional collagen matrices. J Cell Physiol 2004; 200(3): 360-9.

[18] Liu Y, Gan L, Carlsson DJ, et al. A simple, cross-linked collagen tissue substitute for corneal implantation. Invest Ophthalmol Vis Sci 2006; 47(5): 1869-75.

[19] McDonald SM, Matheson LA, McBane JE, et al. Use of monocyte/endothelial cell co-cultures (in vitro) and a subcutaneous implant mouse model (in vivo) to evaluate a degradable polar hydrophobic ionic polyurethane. J Cell Biochem 2011; 112(12): 3762-72.

[20] Kuraitis D, Zhang P, Zhang Y, et al. A stromal cell-derived factor1 releasing matrix enhances the progenitor cell response and blood vessel growth in ischaemic skeletal muscle. Eur Cell Mater 2011; 22: 109-23.

[21] Tønnesen HH, Karlsen J. Alginate in drug delivery systems. Drug Dev Ind Pharm 2002; 28(6): 621-30.

[22] Al-Suwayeh SA, El-Helw AR, Al-Mesned AF, Bayomi MA, ElGorashi AS. In vitro--in vivo evaluation of tableted caseinchitosan microspheres containing diltiazem hydrochloride. Boll Chim Farm 2003;142(1):14-20.

[23] Elcin YM, Dixit V, Gitnick G. Controlled release of endothelial cell growth factor from chitosan-albumin microspheres for localized angiogenesis: in vitro and in vivo studies. Artif Cells Blood Substit Immobil Biotechnol 1996; 24(3): 257-71.

[24] Jain D, Panda AK, Majumdar DK. Eudragit S100 entrapped insulin microspheres for oral delivery. AAPS PharmSciTech 2005; 6(1): E100-7.

[25] Cote MF, Laroche G, Gagnon E, Chevallier P, Doillon CJ. Denatured collagen as support for a FGF-2 delivery system: physicochemical characterizations and in vitro release kinetics and bioactivity. Biomaterials 2004; 25(17): 3761-72.

[26] Taguchi T, Kishida A, Sakamoto N, Akashi M. Preparation of a novel functional hydrogel consisting of sulfated glucoside-bearing polymer: activation of basic fibroblast growth factor. J Biomed Mater Res 1998; 41(3): 386-91.

[27] Berscht PC, Nies B, Liebendorfer A, Kreuter J. Incorporation of basic fibroblast growth factor into methylpyrrolidinone chitosan fleeces and determination of the in vitro release characteristics. Biomaterials 1994; 15(8): 593-600.

[28] Seeger FH, Rasper T, Koyanagi M, Fox H, Zeiher AM, Dimmeler S. CXCR4 expression determines functional activity of bone marrow-derived mononuclear cells for therapeutic neovascularization in acute ischemia. Arterioscler Thromb Vasc Biol 2009;29(11):1802-9.

[29] Fontaine V, Filipe C, Werner N, et al. Essential role of bone marrow fibroblast growth factor- 2 in the effect of estradiol on reendothelialization and endothelial progenitor cell mobilization. Am J Pathol 2006; 169(5): 1855-62.

[30] Kuraitis D, Hou C, Zhang Y, et al. Ex vivo generation of a highly potent population of circulating angiogenic cells using a collagen matrix. J Mol Cell Cardiol 2011; 51(2): 187-97.

[31] Suuronen EJ, Zhang P, Kuraitis D, et al. An acellular matrix-bound ligand enhances the mobilization, recruitment and therapeutic effects of circulating progenitor cells in a hindlimb ischemia model. FASEB J 2009; 23(5): 1447-58.

[32] Kaully T, Kaufman-Francis K, Lesman A, Levenberg S. Vascularization--the conduit to viable engineered tissues. Tissue Eng Part B Rev 2009; 15(2): 159-69.

(C) Kuraitis et al.; Licensee Bentham Open.

This is an open access article licensed under the terms of the Creative Commons Attribution Non-Commercial License (http: //creativecommons.org/licenses/bync/3.0/), which permits unrestricted, non-commercial use, distribution and reproduction in any medium, provided the work is properly cited. 